\title{
ARTICLE \\ Prefrontal cortex response to drug cues, craving, and current depressive symptoms are associated with treatment outcomes in methadone-maintained patients
}

\author{
Andrew S. Huhn ${ }^{1}$, Mary M. Sweeney (D) ${ }^{1}$, Robert K. Brooner ${ }^{1}$, Michael S. Kidorf ${ }^{1}$, D. Andrew Tompkins ${ }^{1,2}$, Hasan Ayaz ${ }^{3,4,5}$ and \\ Kelly E. Dunn ${ }^{1}$
}

Methadone maintenance is an effective treatment for opioid use disorder, yet many methadone-maintained patients (MMPs) continue to struggle with chronic relapse. The current study evaluated whether functional near-infrared spectroscopy (fNIRS) could identify prefrontal cortex (PFC) markers of ongoing opioid use in MMPs, and whether clinical measures of depression and selfreport measures of craving would also be associated with opioid use. MMPs $(n=29)$ underwent a drug cue reactivity paradigm during fNIRS measurements of PFC reactivity. Self-reported opioid craving (measured by a visual analog scale; 0-100) was collected before and after drug cue reactivity, and depressive symptoms were assessed via the 17-item Hamilton Depression Rating Scale (HAM-D). Hierarchical regression and partial correlations were used to evaluate associations between weekly urine drug screens over a 90-day follow-up period and fNIRS, craving, and HAM-D assessments. Neural response to drug cues in the left lateral PFC, controlling for age, sex, and days in treatment was significantly associated with percent opioid-negative urine screens during follow-up $\left(\Delta F_{1,24}=13.19, p=0.001, \Delta R^{2}=0.30\right)$, and correctly classified $86 \%$ of MMPs as either using opioids, or abstaining from opioids $\left(X^{2}(4)=16.28, p=0.003\right)$. Baseline craving $(p<0.001)$ and HAM-D assessment $(p<0.01)$ were also associated with percent opioid-negative urine screens. Combining fNIRS results, baseline craving scores, and HAM-D scores created a robust predictive model $\left(\Delta F_{3,22}=16.75, p<0.001, \Delta R^{2}=0.59\right)$. These data provide preliminary evidence that the fNIRS technology may have value as an objective measure of treatment outcomes within outpatient methadone clinics. Depressive symptoms and drug craving were also correlated with opioid use in MMPs.

Neuropsychopharmacology (2019) 44:826-833; https://doi.org/10.1038/s41386-018-0252-0

\section{INTRODUCTION}

Methadone maintenance is effective in reducing overdose deaths and improving treatment outcomes for persons with opioid use disorder (OUD) [1, 2]. Still, there are major hurdles regarding methadone induction and maintenance [3]. The ability to accurately predict which incoming patients are at highest risk for a negative response to routine care - namely chronic relapse to opioids - could inform clinicians on how to best allocate resources and develop supplementary services to enhance current treatment efforts. Continued opioid use is driven by biological, psychological, and social factors [4]. Therefore, a robust appraisal of treatment outcomes for methadone-maintained patients (MMPs) must take into account human physiology, clinical assessment, and patient feedback.

A common biological factor across substance use disorders (SUDs) is the role of the prefrontal cortex (PFC) in impaired response inhibition and salience attribution, i.e., abnormalities in PFC function are associated with increased drug seeking and use despite negative consequences [5]. Neuroimaging measurements are particularly sensitive in distinguishing group differences in PFC reactivity to drug cues between persons with and without SUDs $[5,6]$. Among individuals with OUD, anatomical changes such as decreased gray matter in the PFC have been further hypothesized to underlie abnormalities in executive function [7]. Functional changes in PFC response to treatment-relevant stimuli have also been observed in persons with OUD. For example, recently withdrawn persons with OUD exhibit increased activation of the left dorsolateral PFC (DLPFC) and/or middle/inferior frontal gyri during drug cue exposure [8], and persons with OUD exhibit increased activation in the adjacent left medial PFC in response to craving memories [9]. A study of MMPs viewing heroin compared to neutral cues found increased activation in the left inferior frontal gyrus and bilateral ventromedial (VM) and DLPFC [10]. It is also important to consider the timing of methadone dose in drug cue reactivity paradigms, as acute methadone exposure reduces neural response to drug compared to neutral cues in the VMPFC and insula [11]. Between-group differences in neural response to drug cues have also been identified in subcortical regions including the bilateral nucleus accumbens and subcallosal cortex of MMPs who relapse versus remain abstinent [12], although no

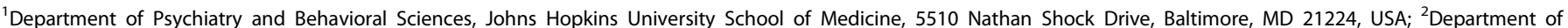

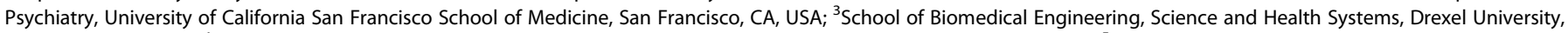

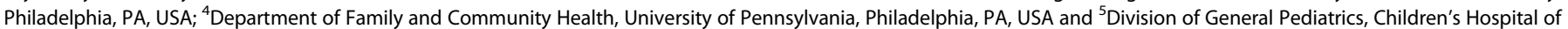
Philadelphia, Philadelphia, PA, USA

Correspondence: Andrew S. Huhn (ahuhn1@jhu.edu)

Received: 30 July 2018 Revised: 13 October 2018 Accepted: 17 October 2018

Published online: 30 October 2018 
neuroimaging studies have examined drug cue reactivity relative to methadone dose as a predictor of treatment outcomes.

In addition to drug cue reactivity, many individuals with OUD experience comorbid major depression, which is associated with negative treatment outcomes relative to individuals with OUD without major depression [13-15]. Furthermore, several studies have shown that depressive symptoms persist after withdrawal from illicit and prescription opioids [16-18], which increases vulnerability to relapse [19]. Similarly, MMPs exhibit high levels of depressive symptoms during maintenance [20, 21]. Low positive affect and high negative affect are associated with craving during OUD recovery $[16,22]$. As a result, craving reduction is an important target for OUD treatment and persistent and/or cueelicited craving is thought to have prognostic value in MMPs [23], although utilizing self-report measures of craving to predict treatment outcomes in OUD patients has been challenging [24].

Previous neuroimaging studies aimed at predicting treatment outcomes for individuals with SUDs have relied heavily on functional magnetic resonance imaging (fMRI) $[6,12,25,26]$, yet the real-world clinical application of fMRI is limited by high cost coupled with the reality that the vast majority of addiction treatment programs exist apart from brain-imaging centers. In order to use neuroimaging as a clinical tool, there is a pressing need to develop technologies that are easy to use, cost effective, and portable. Functional near-infrared spectroscopy (fNIRS) is a neuroimaging tool that monitors hemodynamic response in the PFC, analogous to the blood oxygen level-dependent response measured in fMRI [27]. Moreover, fNIRS is portable, battery operated and wireless, and could be easily deployed in residential or outpatient treatment facilities. The current study evaluated the clinical utility of fNIRS to predict continued opioid use in MMPs. Our hypothesis was that fNIRS measurements of the PFC during a brief, clinic-based, drug cue reactivity paradigm would be associated with 90-day opioid use in MMPs. Moreover, we hypothesized that clinical measures of depressive symptoms and self-report measures of drug craving would also be associated with drug use.

\section{METHODS}

Participants

MMPs were recruited from an outpatient opioid treatment program in Baltimore, MD from September to November 2017. Of the 30 MMPs recruited for this study, one participant was excluded for excessive head motion during the fNIRS session, resulting in a study sample of $N=29$. MMPs were included in the study if they (1) were enrolled in methadone maintenance treatment for OUD, (2) age 18 years or older, (3) were right handed, (4) passed an intoxication test at the time of their methadone dose and study session, which consisted of behavioral, verbal and pupillary observation rated by a registered nurse, and (5) were willing to comply with study protocol including 90-day follow-up (assessed via routine chart review). Exclusion criteria included (1) schizophrenia, bipolar disorder, any psychiatric condition with psychotic features, or dementia, and (2) $<3$ weeks or $>18$ months in methadone treatment or (3) a significant problem, which, in the opinion of the treatment program director, would compromise patient care. The Johns Hopkins Institutional Review Board approved the study protocol and all participants provided informed consent.

\section{Design}

MMPs were scheduled for a single study visit that began immediately after methadone dosing and lasted approximately $3 \mathrm{~h}$. During the visit, participants completed a series of questionnaires including questions about demographics, the Addiction Severity Index [28], and questionnaires related to depressive symptoms (described below). fNIRS testing began approximately
$2 \mathrm{~h}$ after methadone dosing, corresponding to peak effects of methadone [29], and ensuring experimental control over the acute effects of dosing on drug cue reactivity and craving [11]. The fNIRS session included measurements of neural activity in the PFC in response to drug, natural reward, and neutral cues, as well as self-reported ratings of drug craving before and after cue exposure.

\section{Study measures}

Depressive symptoms. Three assessments of depressive symptoms were collected at the study visit. These included the 17-item Hamilton Depression Rating Scale (HAM-D), which assesses past 7day depressive symptoms based on a total summary score [30]. Total HAM-D scores categorize patients as experiencing current depressive symptoms that are normal, mild, moderate, severe, or very severe. The Snaith-Hamilton Pleasure Scale (SHPS), a 14-item questionnaire that measures hedonic tone on a four-point Likert scale [31], was utilized to examine each participant's relative capacity to experience pleasure from natural rewards including clinically meaningful anhedonia (as evidenced by a score $\geq 3$ ); results were summed into a total score. The third assessment was the Demoralization Scale II (DS-II), a 16-item measure of demoralization (defined as a deprivation of morale or an existential challenge with independent components relating to meaning and purpose and distress and coping ability). The Demoralization scale utilizes a three-point Likert scale; results were summed into a total score, as well as distress and coping and meaning and purpose subscales [32].

Craving measures. Craving scales were collected at baseline (e.g., baseline) or following a cue reactivity task (e.g., cue elicited). The following three craving scores were self-reported and measured on a 100-point visual analog rating scale; (1) "How much do you want to use right now?", (2) "How much do you want to avoid using right now?", and (3) "How much control do you feel you have over using right now". Scales were considered to represent the following craving domains: (1) Wanting (higher scores correspond to higher craving), (2) Avoidance (lower scores correspond to higher craving), and (3) Control (lower scores correspond to higher craving).

Cue reactivity task. Following questionnaire completion, a blockdesign, visual cue reactivity paradigm was administered during fNIRS monitoring of the PFC. The cue reactivity paradigm consisted of drug cues, hedonically positive stimuli, and emotionally neutral stimuli. Drug cues were chosen from an internet search of prescription opioids, which were used in lieu of heroin imagery because (1) all participants had a history of prescription opioid use, suggesting that prescription opioids would elicit drug craving, and (2) the outpatient clinic preferred not to utilize heroin imagery. Natural reward and neutral images were sourced from the International Affective Picture System, similar to previous studies $[17,33]$. Participants viewed imagery in an upright, seated position via a 16-inch monitor ( $75 \mathrm{~Hz}$ refresh rate) on which visual cues were delivered via E-Prime software (Psychology Software Tools Inc., PA). Five pictures from a single category (drug, positive reward, neutral) were presented in 25-s blocks (5 pictures/block, each picture displayed for $5 \mathrm{~s}$ ). The order of images within blocks and the order of blocks within the experiment were randomized for each individual and counter-balanced across individuals. Between blocks, a crosshair centered on a black screen was displayed for $10-15 \mathrm{~s}$ to allow hemodynamic flow to return to baseline.

fNIRS measurement

fNIRS measurements were collected during completion of the cue reactivity task. Regional changes in cerebral blood flow (an indirect measure of neural activity) were measured via fNIRS detection of infrared light spectra for oxygenated and 
deoxygenated hemoglobin [34]. In the current study, data were recorded using a continuous wave system (Model 1200, fNIR Devices, LLC, USA) and a pad containing 16 optode (4 LED light sources and 10 photodetectors) and recorded data at $2 \mathrm{~Hz}$ throughout the experimental protocol using COBI Studio [35]. Sensors were aligned at the bottom row of optodes with the International 10-20 sites F7, FP1, FP2, F8 line [36]. This placement situated the sensor over bilateral rostral PFC (Brodmann Area 10) and bilateral ventrolateral/DLPFC [37].

\section{fNIRS signal processing}

fNIRS data processing used raw light intensity from 16 optodes with two wavelengths that were low-pass filtered with a finite impulse response, with a linear phase filter with order 20 and a cutoff frequency of $0.1 \mathrm{~Hz}$ (to attenuate high frequency noise, respiration, and cardiac cycle effects) in Matlab (The Mathworks, Inc., Sherborn, MA) [35]. All data were inspected for potential saturation (defined as light intensity at the detector being greater than the analog-to-digital converter limit) and motion artifact contamination by means of a coefficient of variation based assessment [38]. Data were then corrected for motion artifact, and each task block was extracted and hemodynamic changes for each of the 16 optodes were calculated separately for each block of drug, positive reward, or neutral cues using the Modified Beer Lambert Law. Output consisted of mean deoxygenated hemoglobin $(\mathrm{HbR})$, mean oxygenated hemoglobin $\left(\mathrm{HbO}_{2}\right)$, and mean total hemoglobin (total $\mathrm{Hb}$ ). $\mathrm{HbO}_{2}$ change was extracted for the duration of each 25-s block (drug cue, positive, and neutral) with baseline correction to the beginning of the block for local baseline approach. Analyses in this study were calculated using mean $\mathrm{HbO}_{2}$.

\section{Treatment outcomes}

Continued opioid and other illicit drug use was assessed via weekly urinalysis results for 90 days following the experimental study visit according to clinic electronic health records. All urine specimens were collected via direct observation and subject to temperature testing to reduce falsified specimens. The primary study outcome was continued opioid use within the 90-day monitoring period. The secondary outcome was continued use of any illicit substance, including opioids and also cocaine, benzodiazepines, marijuana, and amphetamines. Opioid or any drug use was treated as a binary (any positive urine drug screen) and continuous (e.g., percent negative urine drug screens) variable during the 90-day follow-up period. One patient who dropped out of treatment during that period was included in the analyses as having continued opioids and any illicit substance use. Baseline drug use was assessed via the addiction severity index and urine drug screens in the 4 weeks leading up to the study session.

\section{Data analysis}

MMPs $(N=29)$ were dichotomized into Any Opioid Use $(n=12)$ or Abstinent $(n=17)$ groups based upon provision of $\geq 1$ opioidpositive urine samples during the 90-day monitoring period. Demographic and questionnaire data were then compared between the groups using independent Student's $t$-tests for continuous or chi-square analysis for dichotomous variables. Given that age, sex, and days in treatment were significantly different between MMPs who used any opioids versus those who abstained, all further analyses on primary and secondary treatment outcomes controlled for these variables. Partial correlations, controlling for age, sex, and days in treatment were used to examine associations between baseline and cue-induced craving, depressive symptoms, and percent negative opioid and illicit drug screens. fNIRS data were considered significant if $\geq 3$ optodes were clustered and correlated to opioid use at $p<0.01$. Hierarchical regression was used to analyze the unique contribution of change in mean $\mathrm{HbO}_{2}$ in response to drug cues as a predictor of percent negative opioid drug screens, including the control variables age, sex, and days in treatment in model 1, and neural response to drug cues in model 2. Given that the change in $\Delta R^{2}$ was significant in this model, a second hierarchical multiple regression was performed, including control variables in model 1 and the strongest predictors across the domains PFC activity, depressive symptoms, and self-reported craving (namely left dorsolateral/ ventrolateral [hereafter referred to as left lateral] PFC response to drug cues, HAM-D scores, and baseline drug Wanting). PFC activity was also used to predict treatment outcome (any opioid use) as a binary variable via logistic regression, controlling for age, sex, and days in treatment. Hierarchical and logistic regression models were also performed on a subgroup of participants who were in treatment for fewer than 4 months $(n=19)$, to examine whether PFC response to drug cues was associated with continued opioid use in early recovery. Alpha levels for partial correlations were Bonferroni corrected to $p<0.008$ for craving scales and $p<0.017$ for depressive symptoms scales. Unless stated otherwise, alpha levels for all other significant findings were set at $p<0.05$. Statistical analyses were conducted using SPSS version 24.0 (IBM, Armonk, NY).

\section{RESULTS}

Participant characteristics

MMPs included in these analyses $(N=29)$ had a mean (SD) age of 42.1 (13.5) years, were $58.6 \%$ male, and $86.2 \%$ white (Table 1 ). In the 4 weeks leading up to the study session, $51.7 \%$ of MMPs provided at least one positive urine drug screen for opioids and $62.1 \%$ provided at least one positive urine drug screen for any illicit substance; during the 90-day follow-up period, $41.4 \%$ of MMPs provided at least one positive urine drug screen for opioids and $58.6 \%$ provided at least one positive urine drug screen for any illicit substance (Table 1).

\section{Depressive symptoms and treatment outcomes}

Mean score for depressive symptoms on the HAM-D for the overall sample was 10.3 ( $S D=8.4$ ) (Table 1). Though HAM-D scores were not significantly different between the Any Opioid Use and Abstinent groups (Table 1), MMPs who used any substance (a secondary treatment outcome) during the 90-day follow-up period had significantly higher scores on the HAM-D $(M=13.2, S D=9.0)$ than patients who abstained $(M=6.0, S D=5.2 ; t(27)=-2.48$, $p=0.02$ ). At the experimental session, $24 \%$ of MMPs reported a severe or very severe level of depressive symptoms, as indicated by a HAM-D score $\geq 19$. MMPs with severe or very severe depressive symptoms were more likely to use any illicit substance (100\%) than MMPs with normal, mild, or moderate levels of depressive symptoms $\left(45.4 \% ; X^{2}(1)=6.51, p=0.01\right)$, including cocaine $(100$ versus $31.8 \%$, respectively; $\left.X^{2}(1)=9.89, p=0.002\right)$, but were not significantly more likely to use opioids (57.1 versus $36.4 \%$, respectively; $x^{2}(1)=0.95, p=0.33$ ). Higher HAM-D scores were significantly associated with lower percent opioid-negative urine drug screens and lower percent illicit drug-negative urine screens during the 90-day follow-up period (Table 2).

On the SHPS, 31\% of MMPs endorsed abnormal hedonic tone at a level indicative of anhedonia. Although there were no significant group differences in SHPS scores between the Any Opioid Use and Abstinent groups, higher SHPS scores were significantly associated with lower percent illicit drug-negative urine screens during the 90-day follow-up period (Table 2).

MMPs in the Any Opioid Use $(M=12.3, S D=8.6)$ compared with the Abstinent $(M=6.7, S D=6.9)$ group reported marginally higher scores on the DS-II $(t(27)=-2.0, p=0.06)$; on the Distress and Coping subscale, MMPs in the Any Opioid Use group $(M=8.1$, $\mathrm{SD}=4.7)$ reported significantly higher scores compared with MMPs in the Abstinent group $(M=4.5, S D=4.4 ; t(27)=-2.1$, $p=0.04$ ) (Table 1). MMPs who used any illicit substance compared with those who abstained reported significantly higher scores on 
Table 1. Participant characteristics

\begin{tabular}{|c|c|c|c|c|}
\hline & All MMPs $(N=29)$ & Any opioid use $(n=12)$ & Abstain from opioids $(n=17)$ & $t$ or $x^{2}$ ( $p$-value $)$ \\
\hline Sex (\% male) & 58.6 & 83.3 & 41.2 & $5.15(0.02)$ \\
\hline Ethnicity (\% Hispanic) & 3.4 & 0 & 5.9 & $0.73(0.39)$ \\
\hline Years education $M(S D)$ & $12.5(2.8)$ & $11.4(2.6)$ & $13.2(2.8)$ & $1.73(0.10)$ \\
\hline Days in treatment $M(S D)$ & $125.9(75.8)$ & $99.5(125.4)$ & $239.7(199.7)$ & $2.15(0.04)$ \\
\hline Past 30-day heroin use $M(S D)$ & $2.8(6.8)$ & $6.3(9.7)$ & $0.3(0.8)$ & $2.13(0.06)$ \\
\hline Past 30-day prescription opioid use M (SD) & $0.6(1.9)$ & $1.1(0.6)$ & $0.0(0.0)$ & $-1.85(0.13)$ \\
\hline Lifetime heroin use $M(S D)$ & $6.3(7.9)$ & $8.3(8.4)$ & $4.9(7.6)$ & $-1.13(0.27)$ \\
\hline Lifetime prescription opioid use M (SD) & $6.7(7.1)$ & $5.3(7.0)$ & $7.7(7.1)$ & $0.89(0.38)$ \\
\hline Gabapentin & 13.8 & 16.7 & 11.8 & $0.14(0.71)$ \\
\hline Ham-D total score M (SD) & $10.3(8.4)$ & $13.3(8.7)$ & $8.1(7.7)$ & $-1.67(0.11)$ \\
\hline SHPS total score M (SD) & $1.7(2.0)$ & $2.3(2.4)$ & $1.4(1.7)$ & $-1.02(0.32)$ \\
\hline Demoralization scale II total score M (SD) & $9.0(8.1)$ & $12.3(8.7)$ & $6.7(6.9)$ & $-1.96(0.06)$ \\
\hline Meaning and purpose subscore & $3.0(3.6)$ & $4.3(4.2)$ & $2.2(2.9)$ & $-1.56(0.13)$ \\
\hline Distress and coping subscore & $6.0(4.8)$ & $8.1(4.7)$ & $4.5(4.4)$ & $-2.14(0.04)$ \\
\hline \multicolumn{5}{|c|}{$\begin{array}{l}\text { Demographics and self-report/clinical assessments of depressive symptoms in methadone-maintained patients who had any opioid use versus those who } \\
\text { remained abstinent during the } 90 \text {-day follow-up period. Group comparisons utilized independent } t \text {-tests for continuous variables and chi-squared for discrete } \\
\text { variables. Significant differences in bold } \\
\text { MMPs methadone-maintained patients, M mean, SD standard deviation, CNS central nervous system, SSRI selective serotonin reuptake inhibitor, HAM-D } \\
\text { Hamilton Depression Scale, SHPS Snaith-Hamilton Pleasure Scale, Demoralization Scale II }\end{array}$} \\
\hline
\end{tabular}

Table 2. Associations between craving, depressive symptoms, and outcomes

\begin{tabular}{|c|c|c|c|c|c|c|c|c|c|}
\hline$\%$ Illicit drug-negative samples & -0.60 & 0.44 & 0.48 & -0.51 & 0.53 & 0.42 & -0.65 & -0.51 & -0.61 \\
\hline HAM-D & 0.46 & 0.05 & -0.79 & 0.52 & -0.39 & -0.67 & 1 & -0.65 & 0.78 \\
\hline SHPS & 0.26 & -0.13 & -0.15 & 0.34 & 0.10 & -0.20 & 0.40 & 1 & 0.54 \\
\hline
\end{tabular}

Partial correlations ( $r$ values) between baseline craving, cue-elicited craving, Hamilton Depression Scale (HAM-D), Snaith-Hamilton Pleasure Scale (SHPS), Demoralization Scale II (DS-II), and percent of urine samples testing negative for opioids and/or any illicit substance (defined as opioid, cocaine, benzodiazepines, marijuana, amphetamines) during the 90-day follow-up period. Wanting refers to desire to use opioids, Avoidance refers to desire to avoid using opioids, and Control refers to perceived control over opioid use. All correlations with relapse to opioids or relapse to any substance controlled for age, sex, and days in treatment. Correlations for craving scales considered significant at $p<0.008$, and correlations for depressive symptom scales considered significant at $p<0.017$ (Bonferroni correction for multiple comparisons). Significant findings in bold

the $\mathrm{DS}-\mathrm{II}(\mathrm{M}=11.9, \mathrm{SD}=8.3$ versus $\mathrm{M}=4.8, \mathrm{SD}=5.7$, respectively; $t(27)=-2.56, p=0.02)$, and on the Distress and Coping subscale $(\mathrm{M}=8.0, \mathrm{SD}=4.8$ versus $\mathrm{M}=3.1, \mathrm{SD}=3.0 ; t(27)=-3.14, p=$ 0.004). Higher DS-II scores were significantly associated with lower percent negative opioid drug screens and percent negative illicit drug screens during the 90-day follow-up period (Table 2).

Craving and treatment outcomes

Higher baseline ratings of craving based on Wanting were significantly associated with lower percent opioid-negative urine drug screens and percent illicit drug-negative urine screens during the 90-day follow-up period (Table 2). In addition, cue-elicited ratings of craving for Avoidance were significantly associated with percent illicit drug-negative urine screens at 90 days (Table 2). Craving scores based on Control were not associated with treatment outcomes. Several measures of craving were also associated with measures of depressive symptoms as shown in Table 2.

fNIRS association with treatment outcomes

The Any Opioid Use group displayed a trend toward increased neural response to drug cues in the left lateral PFC relative to the 


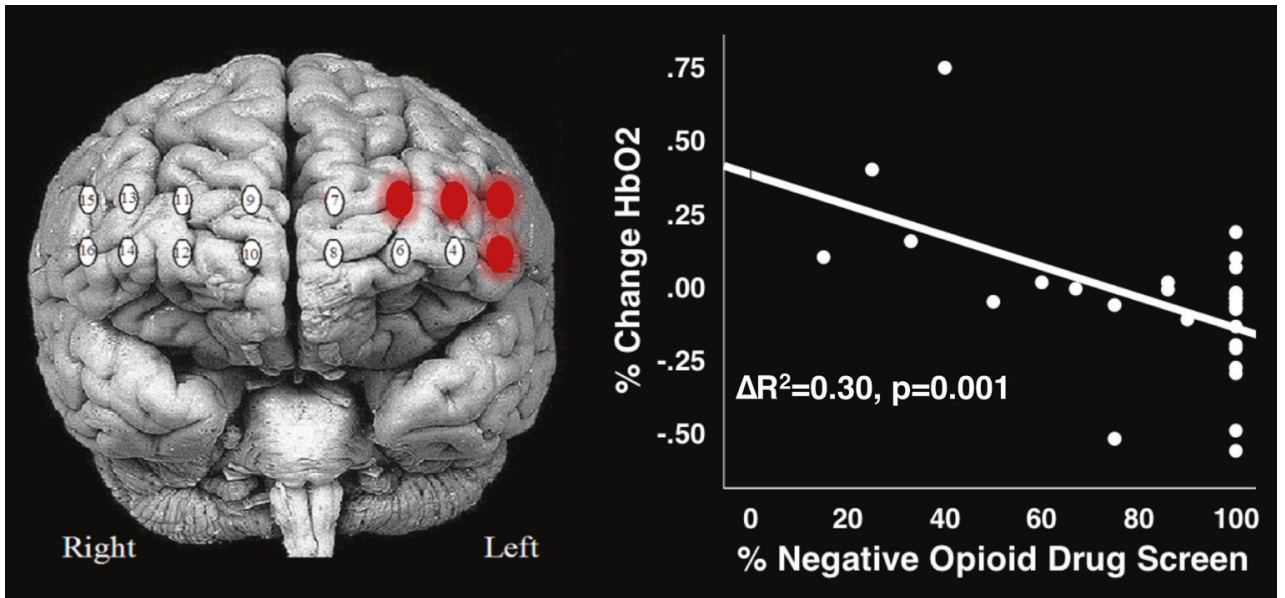

Fig. 1 Association between functional near-infrared spectroscopy measures of deoxygenated hemoglobin in response to opioid cues and percent opioid-negative urine drug screens during 90-day follow-up in methadone-maintained patients. Hierarchical regression model controlled for age, sex, and days in treatment in model 1, and included neural response in the left lateral prefrontal cortex (PFC) in model 2. The total model was significant $\left(F_{4,23}=5.02, p=0.004, R^{2}=0.46\right)$, with a unique contribution from the left lateral PFC response $\left(\Delta F_{1,24}=\right.$ $\left.13.19, p=0.001, \Delta R^{2}=0.30\right)$

Table 3. Hierarchical multiple regression

\begin{tabular}{|c|c|c|c|c|c|c|}
\hline \multirow[b]{2}{*}{ Variables } & \multicolumn{3}{|l|}{ Model 1} & \multicolumn{3}{|l|}{ Model 2} \\
\hline & $B$ & SE $B$ & $\beta$ & $B$ & SE $B$ & $\beta$ \\
\hline Sex & -16.68 & 12.0 & -0.32 & -16.56 & 7.23 & $-0.32 *$ \\
\hline Age & -0.17 & 0.4 & -0.09 & 0.17 & 0.26 & 0.08 \\
\hline Days in treatment & 0.01 & 0.03 & 0.06 & 0.01 & 0.02 & 0.07 \\
\hline $\begin{array}{l}\text { Lateral PFC } \\
\text { response to drug } \\
\text { cue }\end{array}$ & & & & -45.14 & 11.85 & $-0.44^{* *}$ \\
\hline HAM-D & & & & -0.30 & 0.41 & -0.96 \\
\hline Baseline craving & & & & -0.55 & 0.14 & $-0.53 * * *$ \\
\hline$R^{2}$ & 0.16 & & & $0.74 * * *$ & & \\
\hline Adjusted $R^{2}$ & 0.06 & & & 0.67 & & \\
\hline$F$ & 1.56 & & & $10.62 * * *$ & & \\
\hline$\Delta R^{2}$ & & & & 0.59 & & \\
\hline$\Delta F$ & & & & $16.75^{* * *}$ & & \\
\hline
\end{tabular}

Hierarchical multiple regression analysis predicting percent opioidnegative urine drug screens during the 90-day follow-up period Significant findings in bold: ${ }^{*} p<0.05,{ }^{* *} p<0.01,{ }^{* *} p<0.001$

Abstinent group $(t(27)=-2.0, p=0.06)$. Hierarchical linear regression revealed that increased neural response to drug cues in the left lateral PFC was significantly associated with decreased percent opioid-negative urine drug screens during the 90-day follow-up period $\left(F_{4,23}=5.02, p=0.004, R^{2}=0.46\right)$, and left lateral PFC response was a unique contributor to this model $\left(\Delta F_{1,24}=\right.$ 13.19, $p=0.001, \Delta R^{2}=0.30$ ) (Fig. 1). Hierarchical multiple linear regression of neural response in the left lateral PFC, HAM-D total score, and baseline Wanting significantly predicted percent opioid-negative urine drug screens during the 90-day follow-up period $\left(F_{6,22}=10.62, p<0.001, R^{2}=0.74\right)$, and predictor variables were unique contributors to the model $\left(\Delta F_{3,22}=16.75, p<0.001\right.$, $\Delta R^{2}=0.59$ ) (Table 3). Hierarchical regression was also performed on a subgroup of patients who were in treatment for fewer than 4 months $(n=19)$ to determine whether fNIRS measurements could predict continued opioid use among individuals in early recovery. Neural response to drug cues in the left lateral PFC was associated with percent negative opioid drug screens in this subgroup, explaining unique variance over the control variables age, sex, and days in treatment $\left(\Delta F_{1,14}=5.82, p=0.03\right.$, $\Delta R^{2}=0.28$ ).

Logistic regression further suggested that neural response in the left lateral PFC was associated with any opioid use as a binary variable $\left(x^{2}(1)=5.77, p=0.016\right)$; the model including controlling variables was significant $\left(X^{2}(4)=16.28, p=0.003\right)$, explaining $57.9 \%$ (Nagelkerke $R^{2}$ ) of the variance in opioid use and correctly classifying $86.2 \%$ of cases as having either used or abstained from opioids at 90 days (sensitivity $=83.3 \%$, specificity $=88.2 \%$ ). In persons in treatment for fewer than 4 months $(n=19)$, left lateral PFC activity was only marginally associated with any opioid use as a binary variable $\left(X^{2}(1)=2.99, p=0.083\right)$. Left lateral PFC response that predicted use was not correlated with any self-reported ratings of baseline or cue-induced opioid craving and only marginally associated with HAM-D total scores $\left(R^{2}=0.11\right.$, $p=0.09$ ); neural response to natural rewards was not associated with treatment outcomes based on the a priori criteria for fNIRS cluster inclusion.

\section{DISCUSSION}

The current study evaluated objective and subjective predictors of treatment outcomes among MMPs. Study results demonstrated that fNIRS measurement of increased neural activity in the left lateral PFC in response to drug cues was associated with lower percentage of opioid-negative urine drug screens during a 90-day follow-up period (Fig. 1). In addition, left lateral PFC activity correctly classified $86.2 \%$ of MMPs as using or abstaining from opioids during the 90-day follow-up. These data are highly encouraging because neuroimaging markers of treatment outcomes could be developed as an aid in clinical decision-making regarding the course of treatment and allocation of resources for MMPs. Relative to more conventionally evaluated imaging techniques, such as fMRI, fNIRS is a clinic friendly, portable, and easy to use neuroimaging device. Evidence that it can accurately predict treatment outcomes within OUD patients would support its use (1) as an objective treatment outcome assessment tool in OUD treatment settings, and (2) in the development of and testing of specific risk reduction interventions. Response to drug cues in the left lateral PFC was also associated with opioid-negative drug screens in MMPs who were in the early stages of recovery (fewer than 4 months), providing further evidence of the potential benefits of fNIRS in early detection of persons at risk for continued 
illicit opioid use. Moreover, when left lateral PFC responses were evaluated in combination with HAM-D total scores and selfreported baseline Wanting, the predictive power was robust and explained $59 \%$ of the variance in percent opioid-negative urine drug screens during the 90-day follow-up.

A previous $\mathrm{fMRI}$ study conducted among MMPs who did and did not use opioids during a 90-day follow-up reported significant group differences in drug cue reactivity that were relegated to the bilateral nucleus accumbens and subcallosal cortex, although MMPs in that study displayed increased activity in the VM/DLPFC relative to controls [12]. These results differ from the current study, in that group differences in drug cue reactivity were found in subcortical versus cortical brain regions; while the current study found marginal group differences in the lateral PFC between MMPs in the Any Opioid Use versus Abstinent groups during follow-up, hierarchical regression, and logistic regression were utilized to predict opioid use. It is likely that cohort effects and differences in treatment approach affect treatment outcomes in MMPs. It is also likely that subcortical regions known to mediate reward may be significantly associated with treatment outcomes.

Broadly speaking, the DLPFC is involved in substance userelated decision making, allocation of attention, and reward processing [5], and drug cue exposure studies have reported increased activation among persons using nicotine, alcohol, and cocaine who are not in treatment in the left DLPFC and/or middle/ inferior frontal gyri [39-42], and in the bilateral VM/DLPFC and left inferior frontal gyrus of MMPs [10]. Functional variations in subcortical regions such as the nucleus accumbens, caudate, and subthalamic nucleus have also been associated with cue-elicited craving following withdrawal from opioids [8, 43], and similar studies have reported increased activity in the adjacent left medial PFC in response to craving memories [9]. In the current study, we focused on PFC activity that was associated with opioid use and not craving; indeed, the variability in left lateral PFC response was associated with opioid use, and not all participants in this study displayed increased activation to drug versus neutral cues (Fig. 1).

In addition to fNIRS outcomes, the current study found that baseline and cue-elicited craving was independently associated with percent opioid and illicit drug-negative urine screens during the 90-day follow-up period (Table 2). Baseline craving might represent tonic (or persistent) craving that is still present during the peak effects of methadone dose, and cue-elicited craving might model the reaction to drug cues in the natural environment. Although methadone is known to reduce some aspects of general opioid craving [44], MMPs are still prone to opioid use during treatment and it is possible that specific cue-elicited craving may underlie this risk [45]. It is also possible that long-term methadone maintenance may result in reregulation of subcortical regions previously responsible for craving in response to opioid cues [46]; this is supported in part by the fact that MMPs classified as Abstinent in this study tended to have been in treatment longer than MMPs who had Any Opioid Use (Table 1). Moreover, baseline and cue-elicited craving were associated with depressive symptoms (Table 2), consistent with previous studies that examined similar relationships between reduced positive affect, increased negative affect, and craving in persons with OUD [16, 22].

Several domains of depressive symptoms were associated with continued opioid or any illicit substance use in this study. The HAM-D, a clinical assessment of depressive symptoms, was associated with treatment outcomes, as well as baseline and cue-elicited drug Wanting and lack of Control over drug use (Table 2). Individuals with severe or very severe depression appeared to be at significant risk for ongoing drug use; while they were not more likely to use opioids specifically, $100 \%$ of these individuals relapsed to any substance of abuse including cocaine. Ongoing cocaine use is a significant and well-documented issue in MMPs that has been the focus of several independent studies $[47,48]$.
Furthermore, higher scores on the Demoralization Scale II Total Score was associated with treatment outcome and craving measures including cue-elicited Wanting and baseline Control; MMPs classified in the Any Opioid Use group also had higher scores than those in the Abstinent group on the Meaning and Purpose subscale (Table 1). This might be particularly relevant in persons with low socioeconomic status who live in urban areas where drug use is rampant. Finally, the current study extends research on the relationship between anhedonia and OUD by demonstrating that higher levels of anhedonia are associated with continued illicit drug use. Previous neuroimaging research has shown that anhedonia is associated with decreased neural response to positive cues in persons in recovery from OUD $[8,17]$. As predictive models for treatment outcomes become more refined, independent components of depressive symptoms should continue to be evaluated, as various domains of depression might explain unique risk in persons with OUD who also have comorbidities such as major depressive disorder or chronic pain.

It is important to note that reduction of illicit opioid use can be considered a positive treatment outcome for persons on methadone maintenance [49]. The current study conceptualized both reduced opioid use and abstinence from opioid use as positive treatment outcomes. While study results were presented as risk factors for negative outcomes (e.g., more drug use versus any drug use), it is equally important to understand the neuroscience of healthy recovery [50], including the nuance that reduction of illicit substance use reflects a positive step and good therapeutic goal for MMPs, and abstinence from illicit substance use might reflect an ideal goal for long-term, comprehensive recovery.

This study has some limitations. The fNIRS technology does not include an anatomical scan and therefore lacks the spatial resolution of $\mathrm{fMRI}$; however, this study demonstrated that a relatively large area of the left lateral PFC predicted treatment outcome and the downside of reduced spatial resolution might be outweighed by the upside of practicality and clinical utility. In addition, the sample size was relatively small, though consistent with similar imaging-based predictive studies $[6,12,26]$. Another limitation is that MMPs in the Any Opioid Use and Abstinent groups had been in treatment for different lengths of time; however, this is also consistent with other studies $[11,12]$ and is mitigated by the fact that outcomes were significant despite controlling for this variable - which suggests it did not drive study results.

\section{CONCLUSION}

Results from this study suggest that fNIRS response to a drug cue reactivity task, clinical markers of depressive symptoms, and subjective markers of drug craving were independently correlated with 90-day opioid use in MMPs. Furthermore, combining left lateral PFC response to drug cues, HAM-D scores, and selfreported baseline craving created a robust predictive model. These data provide strong preliminary evidence that the fNIRS technology may have value as an objective measure of treatment outcomes within outpatient OUD clinics, and that the development and testing of targeted evidence-based interventions to address negative treatment outcomes could advance the use of precision medicine approaches for OUD.

\section{FUNDING AND DISCLOSURE}

The work described in this article was funded by the National Institute on Drug Abuse: NIDA R21 DA035327 (Dunn). Support for ASH was provided by National Institute on Drug Abuse: NIDA T32DA07209. DAT has received medication supplies from Indivior (formerly Reckitt Benckiser Pharmaceuticals) for an investigator- 
initiated study, was site PI for a clinical trial sponsored by Alkermes, and provided consulting services for AstraZeneca and Theravance. HA was involved in the technology development of the brain-imaging instrument manufactured by fNIR Devices, LLC, and owns a minor share of the firm.

\section{ACKNOWLEDGEMENTS}

We thank Kori Kindbom and her clinical research support team for their help in conducting this study, and the Addiction Treatment Services program patients whose participation made it possible.

\section{ADDITIONAL INFORMATION}

Competing interests: The authors declare that the research was conducted in the absence of any commercial or financial relationships that could be construed as a potential conflict of interest. The remaining authors declare no competing interests.

Publisher's note: Springer Nature remains neutral with regard to jurisdictional claims in published maps and institutional affiliations.

\section{REFERENCES}

1. Kimber J, Larney S, Hickman M, Randall D, Degenhardt L. Mortality risk of opioid substitution therapy with methadone versus buprenorphine: a retrospective cohort study. Lancet Psychiatry. 2015;2:901-8.

2. Ball JC, Ross A. The effectiveness of methadone maintenance treatment: patients, programs, services, and outcome. Springer Science \& Business Media; New York, NY 2012.

3. Volkow ND, Frieden TR, Hyde PS, Cha SS. Medication-assisted therapies-tackling the opioid-overdose epidemic. N Engl J Med. 2014;370:2063-6.

4. Griffiths M. A 'components' model of addiction within a biopsychosocial framework. J Subst Use. 2005;10:191-7.

5. Goldstein RZ, Volkow ND. Dysfunction of the prefrontal cortex in addiction: neuroimaging findings and clinical implications. Nat Rev Neurosci. 2011;12:652-69.

6. Grüsser SM, Wrase J, Klein S, Hermann D, Smolka MN, Ruf M, et al. Cue-induced activation of the striatum and medial prefrontal cortex is associated with subsequent relapse in abstinent alcoholics. Psychopharmacol (Berl). 2004; 175:296-302

7. Lyoo IK, Pollack MH, Silveri MM, Ahn KH, Diaz Cl, Hwang J, et al. Prefrontal and temporal gray matter density decreases in opiate dependence. Psychopharmacol (Berl). 2006;184:139-44.

8. Zijlstra F, Veltman DJ, Booij J, van den Brink W, Franken IH. Neurobiological substrates of cue-elicited craving and anhedonia in recently abstinent opioiddependent males. Drug Alcohol Depend. 2009;99:183-92.

9. Daglish MR, Weinstein A, Malizia AL, Wilson S, Melichar JK, Britten S, et al. Changes in regional cerebral blood flow elicited by craving memories in abstinent opiate-dependent subjects. Am J Psychiatry. 2001;158:1680-6.

10. Wang W, Li Q, Wang Y, Tian J, Yang W, Li W, et al. Brain fMRI and craving response to heroin-related cues in patients on methadone maintenance treatment. Am J Drug Alcohol Abus. 2011;37:123-30.

11. Langleben DD, Ruparel K, Elman I, Busch-Winokur S, Pratiwadi R, Loughead J, et al. Acute effect of methadone maintenance dose on brain FMRI response to heroin-related cues. Am J Psychiatry. 2008;165:390-4.

12. Li Q, Li W, Wang H, Wang Y, Zhang Y, Zhu J, et al. Predicting subsequent relapse by drug-related cue-induced brain activation in heroin addiction: an event-related functional magnetic resonance imaging study. Addict Biol. 2015;20:968-78.

13. Havard A, Teesson M, Darke S, Ross J. Depression among heroin users: 12-month outcomes from the Australian Treatment Outcome Study (ATOS). J Subst Abus Treat. 2006;30:355-62.

14. Rounsaville BJ, Weissman MM, Crits-Christoph K, Wilber C, Kleber H. Diagnosis and symptoms of depression in opiate addicts: course and relationship to treatment outcome. Arch Gen Psychiatry. 1982;39:151-6.

15. Brooner RK, King VL, Kidorf M, Schmidt CW, Bigelow GE. Psychiatric and substance use comorbidity among treatment-seeking opioid abusers. Arch Gen Psychiatry. 1997;54:71-80.

16. Huhn AS, Harris J, Cleveland HH, Lydon DM, Stankoski D, Cleveland MJ, et al. Ecological momentary assessment of affect and craving in patients in treatment for prescription opioid dependence. Brain Res Bull. 2016a;123:94-101.

17. Huhn A, Meyer R, Harris J, Ayaz H, Deneke E, Stankoski D, et al. Evidence of anhedonia and differential reward processing in prefrontal cortex among postwithdrawal patients with prescription opiate dependence. Brain Res Bull. 2016b;123:102-9.
18. Koob GF. Negative reinforcement in drug addiction: the darkness within. Curr Opin Neurobiol. 2013;23:559-63.

19. Kosten TR, Rounsaville BJ, Kleber HD. A 2.5 year follow-up of treatment retention and reentry among opioid addicts. J Subst Abus Treat. 1986;3:181-9.

20. Peles E, Schreiber S, Naumovsky Y, Adelson M. Depression in methadone maintenance treatment patients: rate and risk factors. J Affect Disord. 2007;99:213-20.

21. Weissman MM, Slobetz F, Prusoff B, Mezritz M, Howard P. Clinical depression among narcotic addicts maintained on methadone in the community. Am J Psychiatry. 1976;133:1434-8.

22. Epstein DH, Willner-Reid J, Vahabzadeh M, Mezghanni M, Lin J, Preston KL. Realtime electronic diary reports of cue exposure and mood in the hours before cocaine and heroin craving and use. Arch Gen Psychiatry. 2009;66:88-94.

23. Tiffany ST, Wray JM. The clinical significance of drug craving. Ann N Y Acad Sci. 2012;1248:1-17

24. Dijkstra BA, De Jong CA, Krabbe PF, van der Staak CP. Prediction of abstinence in opioid-dependent patients. J Addict Med. 2008;2:194-201.

25. Seo D, Lacadie CM, Tuit K, Hong K, Constable RT, Sinha R. Disrupted ventromedial prefrontal function, alcohol craving, and subsequent relapse risk. JAMA Psychiatry. 2013;70:727-39.

26. Brewer JA, Worhunsky PD, Carroll KM, Rounsaville BJ, Potenza MN. Pretreatment brain activation during stroop task is associated with outcomes in cocainedependent patients. Biol Psychiatry. 2008;64:998-1004.

27. Moriguchi Y, Noda T, Nakayashiki K, Takata Y, Setoyama S, Kawasaki S, et al. Validation of brain-derived signals in near-infrared spectroscopy through multivoxel analysis of concurrent functional magnetic resonance imaging. Hum Brain Mapp. 2017;38:5274-91.

28. McLellan A, Cacciola J, Zanis D. The addiction severity index-lite. Center for the Studies on Addiction, University of Pennsylvania/Philadelphia VA Medical Center; 1997.

29. Dale O, Sheffels $P$, Kharasch ED. Bioavailabilities of rectal and oral methadone in healthy subjects. Br J Clin Pharmacol. Philadelphia, PA 2004;58:156-62.

30. Hamilton M. A rating scale for depression. J Neurol Neurosurg Psychiatry. 1960;23:56-62.

31. Snaith R, Hamilton M, Morley S, Humayan A, Hargreaves D, Trigwell P. A scale for the assessment of hedonic tone the Snaith-Hamilton Pleasure Scale. Br J Psychiatry. 1995;167:99-103.

32. Robinson S, Kissane DW, Brooker J, Michael N, Fischer J, Franco M, et al. Refinement and revalidation of the demoralization scale: the DS-II-internal validity. Cancer. 2016;122:2251-9.

33. Bunce SC, Harris JD, Bixler EO, Taylor M, Muelly E, Deneke E, et al. Possible evidence for re-regulation of HPA axis and brain reward systems over time in treatment in prescription opioid-dependent patients. J Addict Med. 2015;9: 53-60.

34. Ferrari M, Quaresima V. A brief review on the history of human functional nearinfrared spectroscopy (fNIRS) development and fields of application. Neuroimage. 2012;63:921-35.

35. Ayaz $H$, Shewokis PA, Curtin A, Izzetoglu M, Izzetoglu K, Onaral B. Using MazeSuite and functional near infrared spectroscopy to study learning in spatial navigation. J Vis Exp. 2011;e3443.

36. Jasoer $\mathrm{H}$. Ten-twenty electrode system of the international federation. Electroencephalogr Clin Neurophysiol. 1958;10:371-5.

37. Okamoto M, Dan H, Sakamoto K, Takeo K, Shimizu K, Kohno S, et al. Threedimensional probabilistic anatomical cranio-cerebral correlation via the international 10-20 system oriented for transcranial functional brain mapping. Neuroimage. 2004;21:99-11.

38. Ayaz $H$, Izzetoglu $M$, Shewokis PA, Onaral B. Sliding-window motion artifact rejection for functional near-infrared spectroscopy. Conf. Proc. IEEE Eng. Med. Biol. Soc. 2010;6567-6570.

39. Garavan H, Pankiewicz J, Bloom A, Cho J, Sperry L, Ross TJ, et al. Cue-induced cocaine craving: neuroanatomical specificity for drug users and drug stimuli. Am J Psychiatry. 2000;157:1789-98

40. George MS, Anton RF, Bloomer C, Teneback C, Drobes DJ, Lorberbaum JP, et al. Activation of prefrontal cortex and anterior thalamus in alcoholic subjects on exposure to alcohol-specific cues. Arch Gen Psychiatry. 2001;58:345-52.

41. Maas LC, Lukas SE, Kaufman MJ, Weiss RD, Daniels SL, Rogers VW, et al. Functional magnetic resonance imaging of human brain activation during cue-induced cocaine craving. Am J Psychiatry. 1998;155:124-6.

42. Wilson SJ, Sayette MA, Fiez JA. Prefrontal responses to drug cues: a neurocognitive analysis. Nat Neurosci. 2004;7:211.

43. Li Q, Wang Y, Zhang Y, Li W, Yang W, Zhu J, et al. Craving correlates with mesolimbic responses to heroin-related cues in short-term abstinence from heroin: an event-related fMRI study. Brain Res. 2012;1469:63-72.

44. Shi J, Zhao L, Epstein DH, Zhang X, Lu L. Long-term methadone maintenance reduces protracted symptoms of heroin abstinence and cue-induced craving in Chinese heroin abusers. Pharmacol Biochem Behav. 2007;87:141-5. 
Prefrontal cortex response to drug cues, craving, and current depressive... AS Huhn et al.

45. Fareed A, Vayalapalli S, Stout S, Casarella J, Drexler K, Bailey SP. Effect of methadone maintenance treatment on heroin craving, a literature review. J Addict Dis. 2010;30:27-38.

46. Wang Y, Wang H, Li W, Zhu J, Gold MS, Zhang D, et al. Reduced responses to heroin-cue-induced craving in the dorsal striatum: effects of long-term methadone maintenance treatment. Neurosci Lett. 2014;581:120-4.

47. Umbricht A, DeFulio A, Winstanley EL, Tompkins DA, Peirce J, Mintzer MZ, et al. Topiramate for cocaine dependence during methadone maintenance treatment: a randomized controlled trial. Drug Alcohol Depend. 2014;140:92-100.

48. Carroll KM, Nich C, Shi JM, Eagan D, Ball SA. Efficacy of disulfiram and twelve step facilitation in cocaine-dependent individuals maintained on methadone: a randomized placebo-controlled trial. Drug Alcohol Depend. 2012;126:224-31.

49. Strain EC, Stitzer ML, Liebson IA, Bigelow GE. Dose-response effects of methadone in the treatment of opioid dependence. Ann Intern Med. 1993;119:23-27.

50. Humphreys K, Bickel WK. Toward a neuroscience of long-term recovery from addiction. JAMA Psychiatry. 2018;75:875-6. 